OPEN ACCESS

Edited by:

Robert Czajkowski,

University of Gdańsk, Poland

Reviewed by:

Yantao Jia,

Institute of Microbiology, Chinese

Academy of Sciences (CAS), China

Huamin Chen

Institute of Plant Protection, Chinese

Academy of Agricultural Sciences

(CAAS), China

${ }^{*}$ Correspondence:

Jianuan Zhou

jianuanzhou@scau.edu.cn

${ }^{t}$ These authors have contributed equally to this work

Specialty section:

This article was submitted to Plant Pathogen Interactions, a section of the journal

Frontiers in Plant Science

Received: 17 December 2021

Accepted: 07 January 2022

Published: 08 February 2022

Citation:

Liu F, Hu M, Zhang Z, Xue Y,

Chen S, Hu A, Zhang L-h and Zhou J (2022) Dickeya Manipulates Multiple Quorum Sensing Systems to Control

Virulence and Collective Behaviors.

Front. Plant Sci. 13:838125.

doi: 10.3389/fp/s.2022.838125

\section{Dickeya Manipulates Multiple Quorum Sensing Systems to Control Virulence and Collective Behaviors}

\author{
Fan Liu', Ming Hut, Zhijia Zhang ${ }^{\dagger}$, Yang Xue ${ }^{\dagger}$, Shanshan Chen, Anqun Hu, \\ Lian-hui Zhang and Jianuan Zhou*
}

Guangdong Laboratory for Lingnan Modern Agriculture, Guangdong Province Key Laboratory of Microbial Signals and Disease Control, Integrative Microbiology Research Center, South China Agricultural University, Guangzhou, China

Soft rot Pectobacteriaceae (SRP), typical of Pectobacterium and Dickeya, are a class of Gram-negative bacterial pathogens that cause devastating diseases on a wide range of crops and ornamental plants worldwide. Quorum sensing (QS) is a cell-cell communication mechanism regulating the expression of specific genes by releasing QS signal molecules associated with cell density, in most cases, involving in the vital process of virulence and infection. In recent years, several types of QS systems have been uncovered in Dickeya pathogens to control diverse biological behaviors, especially bacterial pathogenicity and transkingdom interactions. This review depicts an integral QS regulation network of Dickeya, elaborates in detail the regulation of specific QS system on different biological functions of the pathogens and hosts, aiming at providing a systematic overview of Dickeya pathogenicity and interactions with hosts, and, finally, expects the future prospective of effectively controlling the bacterial soft rot disease caused by Dickeya by quenching the key QS signal.

Keywords: soft rot Pectobacteriaceae, Dickeya, quorum sensing, regulation, virulence

\section{INTRODUCTION}

Soft rot Pectobacteriaceae (SRP) belonging to the genera Pectobacterium and Dickeya (Charkowski et al., 2012) are emerging parasitic pathogens listed in the top ten important bacterial phytopathogens in the world (Mansfield et al., 2012). In addition to causing bacterial soft rot, these pathogens also cause blackleg of potato, stalk rot of maize, and foot rot of rice, resulting in considerable economic damage to vegetable and ornamental plant production worldwide. Previously, Dickeya was grouped into the genus Erwinia containing all plant-pathogenic Enterobacteriaceae, but in 2005, it was reclassified as a new genus Dickeya (Samson et al., 2005). Currently, twelve species are included in the Dickeya genus, including Dickeya dianthicola, Dickeya dadantii, Dickeya zeae, Dickeya chrysanthemi, Dickeya paradisiaca, Dickeya solani, Dickeya aquatica, Dickeya fangzhongdai, Dickeya poaceaephila, Dickeya lacustris, Dickeya undicola, and Dickeya oryzae (Samson et al., 2005; van der Merwe et al., 2010; Brady et al., 2012; Parkinson et al., 2014; van der Wolf et al., 2014).

Diverse phenotypic differentiation and complicated pathogenic mechanisms have been revealed in different $D$. zeae and D. solani strains. Recent studies compared the characteristics of $D$. solani strains isolated from countries with different climate conditions and found higher activities of cell wall degrading enzymes (CWDEs) and virulence in Polish strains than in Finland and Israel 
strains (Golanowska et al., 2017). D. oryzae and D. zeae strains isolated from rice, banana, and ornamental clivia in China showed different types of phytotoxins produced, including the zeamine I and zeamine II specific in some of the D. oryzae strains, as well as in D. solani strains (Zhou et al., 2011, 2015; Cheng et al., 2013; Hellberg et al., 2015), and another toxin produced by $D$. zeae banana strain MS2 but not the MS3 strain (Hu et al., 2018). Some D. zeae strains from different sources infect plant hosts in different ranges (Hu et al., 2018, 2021). Moreover, different structures of virulence factor modulating (VFM)-quorum sensing (QS) signals were implicated between D. dadantii 3937 and D. oryzae EC1 and D. zeae banana strains (Lv et al., 2019). Also, the functions of acyl-homoserine lactone (AHL) signals on the virulence of hosts are different in D. oryzae EC1 and D. zeae MS2 (Feng et al., 2019). The diversity of strains and the complexity of pathogenic mechanisms increase the difficulty of disease prevention and control in fields.

The achievement of the successful infection of Dickeya on plants depends on a complex range of virulence factors, including plant cell wall degrading enzymes (PCWDEs) (Hugouvieux-Cotte-Pattat et al., 2014), lipopolysaccharides (LPS), extracellular polysaccharide (EPS) (Condemine et al., 1992), iron carriers (Expert, 1999), pigment indigoidine, type III secretion system (T3SS) (Yang et al., 2002, 2004; Yap et al., 2005), and cell motility and adhesion associated with plants (Hussain et al., 2008; Chen et al., 2016, 2020). Motility, which is regulated by the AHL-QS signal, is a secondary virulence determinant of Dickeya, whereas pectinases in PCWDEs, which are regulated by the VFM-QS signal, are the primary virulence determinants of Dickeya, participating and dominating the macerating soft rot process of the pathogen in plant tissues. The capacity of synthesizing and secreting pectinases is modulated by complex and interconnected circuits involving multiple regulatory pathways. A recent study in our laboratory indicated that putrescine is a transkingdom communication signal modulating cell motility, biofilm formation, and virulence of D. oryzae EC1 (Shi et al., 2019). At the moment of current research, QS signals including the AHL signal, the VFM signal, and the crucial signal putrescine have been shown to participate in the intraspecific and transkingdom cell-cell communication, regulating the infection and the colonization of Dickeya toward host plants.

\section{$N$-ACYL-HOMOSERINE LACTONES-QUORUM SENSING SIGNAL REGULATES CELL MOTILITY AND BIOFILM FORMATION IN Dickeya}

Various gram-negative plant-pathogenic bacteria have been found to use a QS system dependent on the synthesis and perception of $\mathrm{N}$-acyl-homoserine lactones (AHLs) as diffusible signals for coordinating QS communication. The AHL signalmediated QS system is currently the most representative and most studied population sensing system, which is composed of two conserved categories of proteins in nearly all the sequenced Dickeya (except D. paradisiaca) (Potrykus et al., 2014) and Pectobacterium strains, ExpI (LuxI homolog) and ExpR (LuxR homolog). ExpI is a synthase in diverse Pectobacterium and Dickeya plant pathogens in charge of the synthesis of AHLs, including N-3-oxohexanoyl-homoserine lactone (3OC6-HSL), N-3-oxo-octanoyl-homoserine lactone (3OC8-HSL), N-hexanoyl-homoserine lactone (C6-HSL), and $\mathrm{N}$-decanoyl-homoserine lactone (C10-HSL) in Dickeya (Nasser et al., 1998; Crépin et al., 2012; Feng et al., 2019), and $\mathrm{N}$-octanoyl-homoserine lactone (C8-HSL), and N-3oxo-decanoyl-homoserine lactone (3OC10-HSL) additionally in Pectobacterium (Crépin et al., 2012). ExpR is the AHL signal receptor and a transcriptional regulator modulating the expression of target genes.

In Pectobacterium and Dickeya bacteria, ExpR functions as a repressor in most cases, where ExpR binds to the promoter of DNA and blocks transcription in the absence of AHL, and AHL dissociates ExpR-DNA complexes to release ExpR and initiate transcription (Castang et al., 2006; Feng et al., 2019). For example, in D. oryzae EC1 and D. zeae MS2, the deletion of expI but not $\exp R$ dramatically enhanced bacterial motility, aggregation, and pigment production (Hussain et al., 2008; Zhong, 2014; Feng et al., 2019). ExpR also represses its own transcription with a strong affinity for the $\exp R$ regulatory region (Reverchon et al., 1998; Castang et al., 2006). Such autorepression will be relieved with the increase of AHL signal concentration. Unexpectedly, the inactivation of $\operatorname{expI}$ but not $\exp R$ only affects the expression of pelA and pelB but does not result in visible repercussions on total pectate lyase activity in D. dadantii 3937 (Nasser et al., 1998), which also affects the expression of $\operatorname{prt} X$, prtB, $\operatorname{prt} C$, pelB, pelE, pelL, and some zms genes, and slightly reduces the production of pectinases and cellulases, and maceration on potato slices in D. oryzae EC1 (Hussain et al., 2008; Zhong, 2014). However, ExpR has been demonstrated to function as an activator to specifically interact with the promoters of the five major pectinase encoding genes pelA, pelB, pelC, pelD, and pelE (Nasser et al., 1998), and the expI in D. dadantii 3937 (Reverchon et al., 1998), and the addition of AHL signal dissociates ExpRDNA complexes (Castang et al., 2006). In D. dadantii 3937, $D$. oryzae EC1, and D. zeae MS2, the deletion of $\operatorname{expR}$ has no effect on the virulence and the production of major virulence factors (Castang et al., 2006; Zhong, 2014; Feng et al., 2019). An exception is in $D$. solani that the $\exp R$ mutant reduced the virulence on potato tubers by 2 - to 3-fold compared with the wild-type strain (Potrykus et al., 2014), suggesting a certain difference in the degree of regulation on virulence genes by this system in Dickeya depending on the strain and host plant (Potrykus et al., 2018).

In general, the ExpI-ExpR QS system plays an important role in bacterial motility, biofilm formation, and pigment production but has a limited role in the regulation of virulence traits. This system is also under the control of multiple cross-acting regulatory elements, such as cAMP-CRP and PecS in opposite manners (Figure 1A).

However, the ExpI-ExpR QS system has been shown to be quite critical in Pectobacterium species, which is involved 


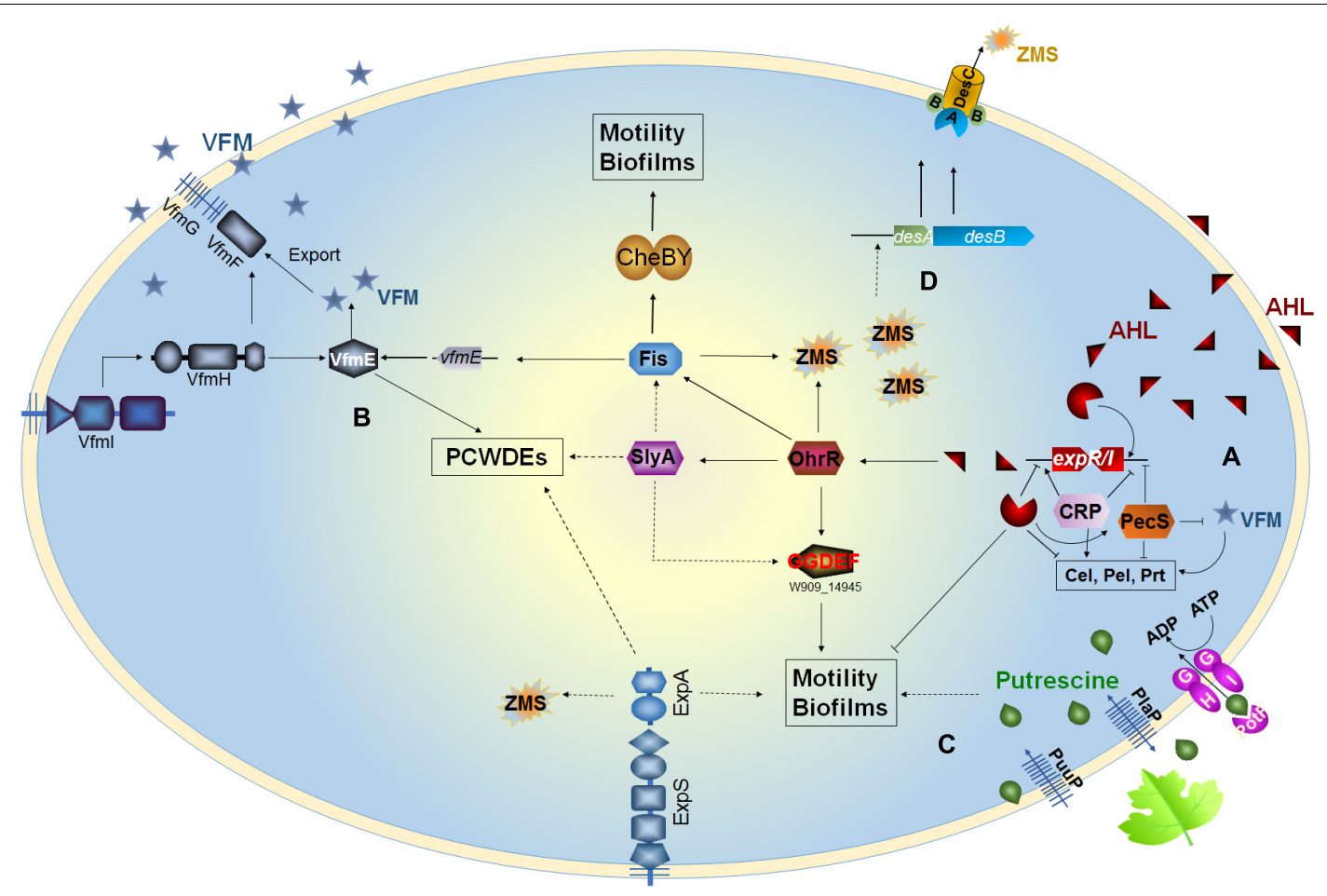

FIGURE 1 | Regulatory pathways of the quorum sensing (QS) systems in Dickeya. (A) Dickeya bacteria produce AHL QS signal to regulate cell motility and biofilm formation; (B) VFM QS signal modulates the production of PCWDEs in Dickeya; (C) Putrescine is a transkingdom communication signal modulating cell motillity and biofilm formation; (D) Zeamines regulate the DesAB efflux pump.

in the production of PCWDEs and other virulence factors in Pectobacterium (Barnard and Salmond, 2007; Liu et al., 2008). There are several virulence regulators in Pectobacterium that act through the Rsm system, which plays a key role in controlling virulence, and the ExpI-ExpR QS system control occupies a vital position in the Pectobacterium regulatory hierarchy, with multiple downstream regulators, such as some that may operate through the Rsm system, also under the ExpI-ExpR QS system control.

Some studies reveal that AHLs produced by bacteria also act as interkingdom signals which influence and reprogram plant gene expression and also interact with other microbes (Mathesius et al., 2003; Bauer and Mathesius, 2004; Schuhegger et al., 2006; OrtÍzCastro et al., 2008; von Rad et al., 2008; Schikora et al., 2011; Schenk et al., 2012).

Apart from the $\operatorname{luxR}$ genes paired with the adjacent luxI genes, many bacteria harbor $\operatorname{luxR}$ genes without $\operatorname{luxI}$ genes in their vicinity on the chromosome, called orphan or solo $\operatorname{luxR}$ (Hudaiberdiev et al., 2015). These solo $\operatorname{luxR}$ may respond to either internal AHL signals such as the $q s c R$ of Pseudomonas aeruginosa (Lequette et al., 2006) or exogenous signals such as the sdiA of Escherichia coli and Salmonella enterica (Ahmer, 2004; Hughes et al., 2010). In D. zeae MS2, the solo luxR $(p i p R)$ is linked to a proline iminopeptidase encoding gene pipA (Feng et al., 2019), similar to the solo luxR homologs in Xanthomonas, Pseudomonas, and Kosakonia (Zhang et al., 2007; Ferluga and Venturi, 2009; Coutinho et al., 2018; Mosquito et al., 2020). The expression of pipA is under the control of PipR, and the deletion of the $p i p A$, but not $p i p R$, in MS2 strain fully impaired its virulence on banana seedlings (Feng et al., 2019). In Dickeya genus, the luxR-solo systems, containing the $p i p A / p i p R$ and the ABC-type peptide transporter genes (Feng et al., 2019), are only present in some strains including D. parazeae Ech586, 6 strains of D. zeae, 2 strains of D. fangzhongdai, D. dadantii, and D. dianthicola, respectively, 6 strains of D. solani, and Dickeya sp. Secpp 1600 (Table 1). Interestingly, this system is highly conserved in many Klebsiella strains but absent in the closely related species of $D$. oryzae (Table 1). Notably, all the host plants of D. parazeae and D. zeae harboring this luxR-solo system belong to monocotyledons (Table 1), suggesting that the system may be related to host specialization.

\section{THE Dickeya-SPECIFIC VIRULENCE FACTOR MODULATING SIGNAL REGULATES PLANT CELL WALL DEGRADING ENZYMES PRODUCTION AND VIRULENCE}

In 2013, Nasser et al. (2013) first identified the existence of a non-AHL-QS system in D. dadantii 3937, called the VFM system. This system is encoded by a $25-\mathrm{kb}$ gene cluster, containing genes involved in the biosynthesis, transport, and induction of the VFM signal. With the exception of D. poaceaephila NCPPB 569 isolated from sugarcane, which had only $46 \%$ coverage, the $v f m$ gene 


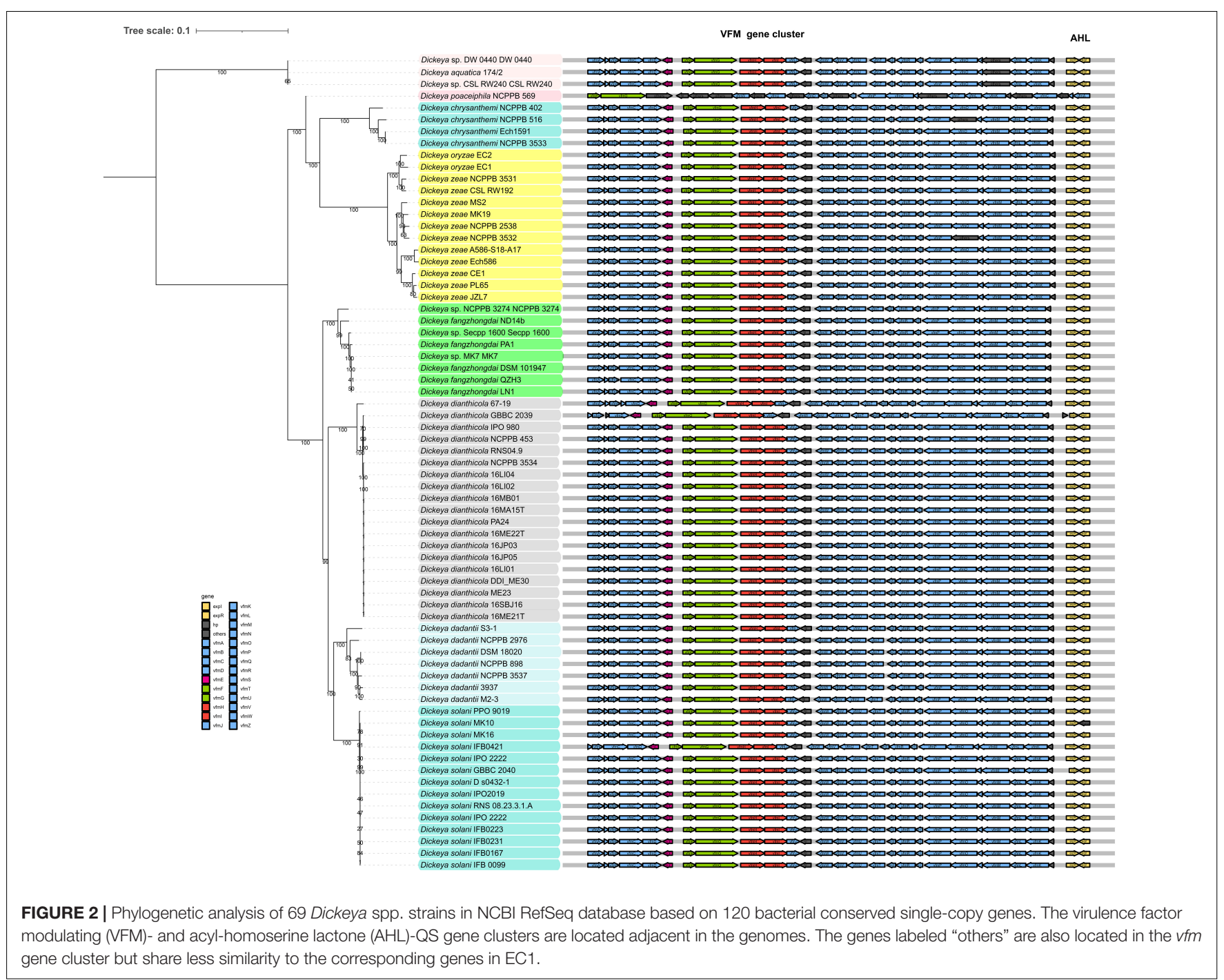

cluster is extremely conserved in all Dickeya strains whose whole genome sequences have been sequenced (Figure 2). Notably, this gene cluster is uniquely present in Dickeya strains, suggesting that the VFM-QS system is an inherent, ubiquitous, and unique regulatory mechanism in Dickeya. In all the tested strains such as $D$. dadantii 3937 , four $D$. solani strains, $D$. oryzae EC1, and D. zeae MS2, the VFM-QS system has been demonstrated to participate in the regulation of PCWDE production and virulence (Nasser et al., 2013; Potrykus et al., 2014, 2018; Lv et al., 2019). Furthermore, in D. oryzae EC1, the VFM signal also regulates pathogen swimming and swarming motility and the production of phytotoxin zeamines (Lv et al., 2019).

Although the chemical structure of the VFM signal has not been characterized yet, some characteristics of this signal can be inferred through some experimental evidence. First, the signal is an extracellular compound since it can be sensed by the VfmI histidine kinase sensor using bacterial supernatant and activates the expression of the $v f m E$, encoding an AraCtype family transcriptional regulator through the VfmHI twocomponent system ( $\mathrm{Lv}$ et al., 2019; Figure 1B). Second, the nature of the VFM signals produced by different Dickeya strains might be different. A lacZ-VFM reporter, constructed based on $D$. oryzae EC1 lacZ deletion mutant, could sense the VFM signals produced by EC1, D. zeae MS2 and MS3, but not strain $D$. dadantii 3937, vice versa ( $\mathrm{Lv}$ et al., 2019), suggesting that the chemical structures of the VFM signals produced by EC1 and 3937 might be different. Moreover, the VFM signal from 3937 is stable after treatment by boiling at $100^{\circ} \mathrm{C}$ for $3 \mathrm{~min}$ (Nasser et al., 2013), while that from EC1 completely loses its activity after treatment at $50^{\circ} \mathrm{C}$ for $30 \mathrm{~min}$ (Lv, 2018). Third, the VFM signal is produced at the early and middle stages of bacterial exponential growth. During the growth of $D$. oryzae EC1, the production of VFM signal was quantified, and the results showed that when the cell density is lower than $\mathrm{OD}_{600}$ at 0.5 , the VFM signal increased slowly with the increase of bacterial concentration; when the cell density is over $\mathrm{OD}_{600}$ at 0.5 , the signal generation rate is obviously accelerated; after $\mathrm{OD}_{600}$ at 1.2 , interestingly, the signal concentration decreased sharply (Lv, 2018), suggesting that VFM signal degrading enzymes are probably present in the 
TABLE 1 | Homologs of the luxR-solo systems (C1030_RS14500 to C1030_RS14535) of Dickeya zeae MS2.

\begin{tabular}{|c|c|c|c|c|}
\hline Strain & Country & Host & Coverage & Identity \\
\hline Dickeya zeae MS2 & Guangzhou & Banana & $100 \%$ & $100 \%$ \\
\hline Dickeya parazeae Ech586 & NA & Philodendron & $100 \%$ & $97.76 \%$ \\
\hline Dickeya zeae A586-S18-A17 & France: Durance River & River water & $100 \%$ & $97.38 \%$ \\
\hline Dickeya zeae JZL7 & China: Guangzhou & Clivia minita & $100 \%$ & $96.24 \%$ \\
\hline Dickeya zeae PL65 & United States: Hawaii & Taro & $100 \%$ & $96.04 \%$ \\
\hline Dickeya zeae A5410 & United States: Hawaii & Pineapple & $100 \%$ & $95.12 \%$ \\
\hline Dickeya zeae CE1 & China: Meizhou & Canna edulis & $98 \%$ & $96.48 \%$ \\
\hline Dickeya fangzhongdai DSM $101947^{\top}$ & Zhejiang & Pyrus pyrifolia & $90 \%$ & $72.52 \%$ \\
\hline Dickeya fangzhongdai ND14b & Malaysia & Waterfall & $90 \%$ & $72.47 \%$ \\
\hline Dickeya solani IFB0223 & Germany & Potato rhizosphere & $90 \%$ & $72.13 \%$ \\
\hline Dickeya solani IFB 0099 & Poland & Potato & $90 \%$ & $72.13 \%$ \\
\hline Dickeya solani PPO 9019 & Netherlands & Muscari & $90 \%$ & $72.13 \%$ \\
\hline Dickeya solani D s0432-1 & Finland & Potato stem & $90 \%$ & $72.13 \%$ \\
\hline Dickeya solani RNS 08.23.3.1.A & France & Potato & $90 \%$ & $72.13 \%$ \\
\hline Dickeya solani IPO $2222^{\top}$ & Netherlands & Potato & $90 \%$ & $72.13 \%$ \\
\hline Dickeya dadantii DSM $18020^{\top}$ & Comoros & Pelargonium capitatum & $90 \%$ & $71.79 \%$ \\
\hline Dickeya dadantii 3937 & NA & Saintpaulia ionantha & $90 \%$ & $71.52 \%$ \\
\hline Dickeya dianthicola ME23 & United States:Maine & Potato & $86 \%$ & $73.08 \%$ \\
\hline Dickeya dianthicola RNS04.9 & NA & Potato & $86 \%$ & $73.08 \%$ \\
\hline Dickeya sp. Secpp 1600 & Hunan & Radish & $90 \%$ & $72.51 \%$ \\
\hline $\begin{array}{l}\text { Klebsiella oxytoca } \\
\text { 4928STDY7071292, } \\
\text { 4928STDY7071186, }\end{array}$ & United Kingdom & Human fecal & $94 \%$ & $79.63 \%$ \\
\hline 4928STDY7387739, & & & & $-79.96 \%$ \\
\hline \multicolumn{5}{|l|}{ 4928STDY7387706, } \\
\hline \multicolumn{5}{|l|}{ 4928STDY7387738 } \\
\hline Klebsiella michiganensis BD177 & NA & NA & $94 \%$ & $79.67 \%$ \\
\hline Klebsiella michiganensis K518, K516 & Zhejiang & Human fecal & $94 \%$ & $79.67 \%$ \\
\hline Klebsiella oxytoca CAV1374 & United States:Virginia & Human perirectal & $94 \%$ & $79.67 \%$ \\
\hline Klebsiella michiganensis E718 & Taiwan & Human & $94 \%$ & $79.67 \%$ \\
\hline Klebsiella michiganensis F107 & Fujian & Human sputum & $94 \%$ & $79.65 \%$ \\
\hline Klebsiella oxytoca $\mathrm{KONIH} 4, \mathrm{KONIH} 2$ & United States & Waste water & $94 \%$ & $\begin{array}{l}79.64 \% \\
79.63 \%\end{array}$ \\
\hline Klebsiella oxytoca KONIH1 & United States & Perirectal swab & $94 \%$ & $79.64 \%$ \\
\hline Klebsiella michiganensis C52 & Sydney & Human clinical sample & $94 \%$ & $79.61 \%$ \\
\hline Klebsiella michiganensis AR375 & NA & NA & $94 \%$ & $79.62 \%$ \\
\hline Klebsiella oxytoca AR_0028 & NA & NA & $94 \%$ & $79.60 \%$ \\
\hline Klebsiella michiganensis M1 & South Korea & Soil & $94 \%$ & $79.61 \%$ \\
\hline Klebsiella michiganensis HKOPL1 & NA & Panda fecal & $94 \%$ & $79.61 \%$ \\
\hline Klebsiella michiganensis KCTC 1686 & South Korea & NA & $94 \%$ & $79.61 \%$ \\
\hline Raoultella electrica DSM 102253 & Japan & Anodic biofilm of microbial fuel cell & $94 \%$ & $79.03 \%$ \\
\hline
\end{tabular}

genome of EC1. In addition, a pecS gene encoding a MarR family transcriptional regulator, along with the indigoidine biosynthesis gene ind $A B C$, is located upstream of the $v \mathrm{fm}$ gene cluster. These genes are mainly present in Dickeya spp. and absent in Pectobacterium spp. PecS functions as a repressor of the VFM system, and the deletion of pecS induces symptoms more rapidly than the wild-type strain (Hommais et al., 2008; Pédron et al., 2018), which may be attributed to the overproduction of VFM signal in the pecS mutant. PecS acts mainly in the early stages of infection, suggesting that it may prevent the $v \mathrm{fm}$ gene expression in the first stages of infection. Furthermore, VFM- and AHL-QS systems do not work in synergy to modulate the virulence of Dickeya. Previous studies have revealed that the AHL-QS system regulates the cell motility and biofilm formation of Dickeya spp., playing an essential role in pathogen colonization and survival in poor environmental conditions (Hussain et al., 2008; Feng et al., 2019), while the VFMQS system is more dominant in regulating the production of diverse virulence factors and the ability to macerate plant tissue (Nasser et al., 2013; Lv et al., 2019). The interplay between these two QS systems has been studied in five D. solani strains at different virulence levels. The results showed that the two QS systems do not coordinate the virulence of Dickeya (Potrykus et al., 2018). 
Apart from the PecS, Fis transcriptional regulator has been found to bind directly to the promoter region of $v f m E$, directly regulating the production of VFM signal and the PCWDEs (Lv et al., 2018). Recently, an organic hydroperoxide reductase regulator (OhrR) has been identified to directly bind to the promoter regions of fis and slyA (Lv et al., 2021). The two transcriptional regulators Fis and SlyA control the production of PCWDEs in completely different pathways (Zhou et al., 2016; Lv et al., 2018).

\section{PUTRESCINE ACTS AS A NEW TYPE OF QUORUM SENSING AND TRANSKINGDOM COMMUNICATION SIGNAL}

Polyamines, mainly constituted by putrescine, spermine, spermidine, and cadaverine, are a group of aliphatic small polycationic molecules that can bind to RNA, DNA, nucleotide triphosphates, and other acidic substances, involved in regulating a wide variety of physiological processes within living organisms (Igarashi and Kashiwagi, 2000; Shi et al., 2019). In recent years, considerable evidence has demonstrated that in addition to core physiological functions, including translation, transcription, and chromatin remodeling, polyamines are involved in various fundamental cellular processes regulated by bacterial cells as QS signals. For instance, norspermidine acts as an intercellular signaling molecule that activates the biofilm formation of Vibrio cholera via a norspermidine sensor NspS-dependent manner, and the lack of norspermidine biosynthetic pathway results in severely reduced biofilm formation (Griffiths et al., 1984; Karatan et al., 2005; Lee et al., 2009). Spermidine transporter-dependent signaling pathway regulates the expression of the T3SS genes of $P$. aeruginosa (Zhou et al., 2007). Putrescine has been demonstrated to be critical for biofilm formation and motility in common human pathogens. In E. coli, putrescine interferes with biofilm formation and surface motility (Koski and Vaara, 1991; Kurihara et al., 2011). In Yersinia pestis, the deficiency of putrescine synthesis enzymes can severely inhibit biofilm formation, and the exogenous addition of putrescine can effectively rescue biofilm formation (Patel et al., 2006; Wortham et al., 2010). In the urinary tract pathogen Proteus mirabilis, putrescine serves as an extracellular signal essential for swarming motility and invasion ability (Sturgill and Rather, 2004; Kurihara et al., 2013).

In Dickeya spp., only putrescine and spermidine are produced since the spermine synthase encoding gene spe 4 is absent in the genomes. The previous study has shown that the deletion of the arginine decarboxylase encoding gene speA in D. oryzae EC1 impaired the synthesis of putrescine, bacterial motility, biofilm formation, and rice seed invasion ability (Shi et al., 2019). Putrescine can also act as an interkingdom communication signal transmitted through the bacterial plasma membrane by PotF and $\mathrm{PlaP}$ putrescine-specific transporters into the wild-type EC1 and speA mutant cells to activate and rescue the phenotype of bacterial motility (Shi et al., 2019; Figure 1C). In D. zeae MS3, the deletion of $s p e A$, but not speE (encoding spermidine synthase), decreases bacterial motility, biofilm formation, and virulence on banana seedlings and potato slices but has no effect on the production of PCWDEs (Tang, 2017), suggesting that putrescine, but not spermidine, is a conserved critical signal regulating the cell motility, biofilm formation, and virulence in Dickeya.

\section{ZEAMINES MODULATE TOXIN PRODUCTION AND TOXIN RESISTANCE IN A MANNER OF QUORUM SENSING MECHANISM}

Zeamines are polyamine phytotoxins produced by $D$. oryzae $\mathrm{EC} 1$, many D. solani, and some Serratia strains (Zhou et al., 2015). Zeamines are encoded by a $z m s$ gene cluster that includes 18 genes from $z m s \mathrm{O}$ to $z m s \mathrm{~N}$ (Zhou et al., 2015). Among them, $z m s \mathrm{~A}$ is a key gene responsible for the biosynthesis of zeamines (Zhou et al., 2011), and $z m s K$ encodes a non-ribosomal peptide synthase (NRPS) catalyzing the amide bond formation by using zeamine II as a substrate to generate zeamine (Cheng et al., 2013). The mutation of $z m s \mathrm{~A}$ and $z m s K$ abolishes antimicrobial activity and attenuates the virulence of D. oryzae EC1 (Zhou et al., 2011; Cheng et al., 2013). Zeamines not only function as virulence factors in D. oryzae rice strains (Zhou et al., 2011; Cheng et al., 2013) but also antagonize many bacteria and fungi and even kill the nematode (Hellberg et al., 2015; Liao et al., 2015; Hu et al., 2018).

In $\mathrm{EC} 1$, the production of zeamines is under the control of multiple regulation pathways, such as the OhrR-SlyA-Fis, the OhrR-SlyA-Fis-VfmE, the VfmI-VfmH-VfmE, and the ExpSExpA (Zhou et al., 2016; Lv et al., 2018, 2019, 2021). Notably, an efflux pump DesABC specifically recognizes zeamines and is currently only found in many Dickeya spp. (Liang et al., 2019), implicating a mechanism of toxin tolerance in Dickeya bacteria. Surprisingly, zeamines also act as a regulator modulating the expression of des $\mathrm{AB}$ genes in a density-dependent manner. The exogenous addition of zeamines in a low concentration $(5 \mu \mathrm{g} / \mathrm{ml})$ could significantly induce the expression of des $\mathrm{AB}$ genes in D. oryzae EC1 and D. dadantii 3937 (Liang et al., 2019; Figure 1D), unveiling a novel and specific signaling role of zeamines in regulating microbial resistance to zeamines.

\section{CONCLUSION}

Quorum sensing is a "language" for microbial communication to regulate the group behavior of microbes. The vital role of QS in bacterial virulence has attracted considerable interest from researchers, making it a promising novel target for the prevention and control of QS-mediated bacterial infections (Joshi et al., 2016). Such novel disease control strategy, called quorum quenching $(\mathrm{QQ})$, is distinguished from other disease biocontrol measures in that QQ disrupts signal-mediated QS by inactivating QS signal or interfering with signal production or perception, 
not acting on the main growth factors of the pathogens; thus, it would not cause selective pressure on the survival of pathogens. In this study, QS systems that regulate the pathogenesis of Dickeya are systematically revealed which not only modulate the virulence of Dickeya but also affect the drug resistance and adaptability to the environment. They are differential in chemical structures, biosynthesis pathways, signal transduction pathways, and regulation mechanisms. For the widely conserved classical AHL-QS system, it mainly affects bacterial motility and biofilm formation and regulates the adaptability of Dickeya spp. to the surrounding environment. Except in D. solani strains that cause blackleg of potato disease in Western Europe, the AHL-QS system regulates the virulence of the pathogens (Potrykus et al., 2018). Given that VFM and putrescine are ubiquitous in Dickeya spp. and they function as major regulatory systems modulating virulence of Dickeya spp., we suggest focusing on quenching these two systems for prevention and control of bacterial soft rot on crops caused by Dickeya.

From current research results, no obvious evidence reveals that there is a relationship between AHL and VFM systems. However, since the VFM signal actions at a relatively low cell density (OD600 below 1.2), while the AHL signal functions when the cell density is high, we think that some gene(s) may be responsible for the switching between these two QS systems. For

\section{REFERENCES}

Ahmer, B. (2004). Cell-to-cell signalling in Escherichia coli and Salmonella enterica. Mol. Microbiol. 52, 933-945. doi: 10.1111/j.1365-2958.2004.04 054.x

Barnard, A. M. L., and Salmond, G. P. C. (2007). Quorum sensing in Erwinia species. Anal. Bioanal. Chem. 387, 415-423. doi: 10.1007/s00216-006-0701-1

Bauer, W. D., and Mathesius, U. (2004). Plant responses to bacterial quorum sensing signals. Curr. Opin. Plant Biol. 7, 429-433. doi: 10.1016/j.pbi.2004. 05.008

Brady, C. L., Cleenwerck, I., Denman, S., Venter, S. N., Rodriguez-Palenzuela, P., Coutinha, T., et al. (2012). Proposal to reclassify Brenneria quercina (Hildebrand and Schroth 1967) Hauben et al. 1999 into a new genus, Lonsdalea gen. Nov., As Lonsdalea quercina comb. Nov., Descriptions of Lonsdalea quercina subsp quercina comb. Nov., Lonsdalea quercina subsp iberica subsp nov and Lonsdalea quercina subsp britannica subsp nov., emendation of the description of the genus Brenneria, reclassification of Dickeya dieffenbachiae as Dickeya dadantii subsp dieffenbachiae comb. Nov., And emendation of the description of Dickeya dadantii. Int. J. Syst. Evol. Micr. 62, 1592-1602. doi: 10.1099/ijs.0.035055-0

Castang, S., Reverchon, S., Gouet, P., and Nasser, W. (2006). Direct evidence for the modulation of the activity of the Erwinia chrysanthemi quorum-sensing regulator ExpR by acylhomoserine lactone pheromone. J. Biol. Chem. 281, 29972-29987. doi: 10.1074/jbc.M601666200

Charkowski, A., Blanco, C., Condemine, G., Expert, D., Franza, T., Hayes, C., et al. (2012). The role of secretion systems and small molecules in Soft-Rot Enterobacteriaceae pathogenicity. Annu. Rev. Phytopathol. 50, 425-449. doi: 10.1146/annurev-phyto-081211-173013

Chen, Y., Lv, M., Liao, L., Gu, Y., Liang, Z., Shi, Z., et al. (2016). Genetic modulation of c-di-GMP turnover affects multiple virulence traits and bacterial virulence in rice pathogen Dickeya zeae. PLoS One 11:e0165979. doi: 10.1371/journal.pone. 0165979

Chen, Y., Zhou, J., Lv, M., Liang, Z., Parsek, M. R., and Zhang, L. H. (2020). Systematic analysis of c-di-GMP signaling mechanisms and biological functions in Dickeya zeae EC1. mBio 11, e02993-20. doi: 10.1128/mBio.02993-20

Cheng, Y., Liu, X., An, S., Chang, C., Zou, Y., Huang, L., et al. (2013). A nonribosomal peptide synthase containing a Stand-Alone condensation the putrescine signal, some of the regulon overlays with those regulated by the AHL signal, but no current evidence indicates any interplay between them. The interactions between different QS systems in Dickeya need more in-depth investigations to draw a more accurate and clear conclusion.

\section{AUTHOR CONTRIBUTIONS}

FL, MH, ZZ, YX, SC, AH, and JZ wrote the manuscript. L-HZ and JZ revised the manuscript. All authors contributed to the article and approved the submitted version.

\section{FUNDING}

This study was financially supported by grants from the National Natural Science Foundation of China (31972230), the Key-Area Research and Development of Guangdong Province (2020B0202090001 and 2018B020205003), the Natural Science Foundation of Guangdong Province, China (2020A1515011534), the Science and Technology Planning Project of Shaoguan City (200805094530618), and the Guangzhou Basic Research Program (202102080613).

domain is essential for phytotoxin zeamine biosynthesis. Mol. Plant Microbe Interact. 26, 1294-1301. doi: 10.1094/MPMI-04-13-0098-R

Condemine, G., Dorel, C., Hugouvieux-Cotte-Pattat, N., and Robert-Baudouy, J. (1992). Some of the out genes involved in the secretion of pectate lyases in Erwinia chrysanthemi are regulated by kdgR. Mol. Microbiol. 6, 3199-3211. doi: 10.1111/j.1365-2958.1992.tb01775.x

Coutinho, B. G., Mevers, E., Schaefer, A. L., Pelletier, D. A., Harwood, C. S., Clardy, J., et al. (2018). A plant-responsive bacterial-signaling system senses an ethanolamine derivative. Proc. Natl. Acad. Sci. U. S. A. 115, 9785-9790. doi: 10.1073/pnas.1809611115

Crépin, A., Beury-Cirou, A., Barbey, C., Farmer, C., Helias, V., Burini, J. F., et al. (2012). N-Acyl homoserine lactones in diverse Pectobacterium and Dickeya plant pathogens: diversity, abundance, and involvement in virulence. Sensors 12, 3484-3497. doi: 10.3390/s120303484

Expert, D. (1999). WITHHOLDING and EXCHANGING IRON: interactions between Erwinia spp. And their plant hosts. Annu. Rev. Phytopathol. 37, 307-334. doi: 10.1146/annurev.phyto.37.1.307

Feng, L., Schaefer, A. L., Hu, M., Chen, R., Greenberg, E. P., and Zhou, J. (2019). Virulence factor identification in the banana pathogen Dickeya zeae MS2. Appl. Environ. Microb. 85, e01611-19. doi: 10.1128/AEM.01611-19

Ferluga, S., and Venturi, V. (2009). OryR is a LuxR-Family Protein Involved in Interkingdom Signaling between Pathogenic Xanthomonas oryzae pv. oryzae and Rice. J. Bacteriol. 191, 890-897. doi: 10.1128/JB.01507-08

Golanowska, M., Kielar, J., and Lojkowska, E. (2017). The effect of temperature on the phenotypic features and the maceration ability of Dickeya solani strains isolated in Finland, Israel and Poland. Eur. J. Plant Pathol. 147, 803-817. doi: 10.1007/s10658-016-1044-1

Griffiths, G. L., Sigel, S. P., Payne, S. M., and Neilands, J. B. (1984). Vibriobactin, a siderophore from Vibrio cholerae. J. Biol. Chem. 259, 383-385. doi: 10.1016/ s0021-9258(17)43671-4

Hellberg, J. E. E. U., Matilla, M. A., and Salmond, G. P. C. (2015). The broadspectrum antibiotic, zeamine, kills the nematode worm Caenorhabditis elegans. Front. Microbiol. 6:137. doi: 10.3389/fmicb.2015.00137

Hommais, F., Oger-Desfeux, C., Van Gijsegem, F., Castang, S., Ligori, S., Expert, D., et al. (2008). PecS is a global regulator of the symptomatic phase in the phytopathogenic bacterium Erwinia chrysanthemi 3937. J. Bacteriol. 190, 7508-7522. doi: 10.1128/JB.00553-08 
Hu, M., Li, C., Xue, Y., Hu, A., Chen, S., Chen, Y., et al. (2021). Isolation, characterization, and genomic investigation of a phytopathogenic strain of Stenotrophomonas maltophilia. Phytopathology 111, 2088-2099. doi: 10.1094/ PHYTO-11-20-0501-R

Hu, M., Li, J., Chen, R., Li, W., Feng, L., Shi, L., et al. (2018). Dickeya zeae strains isolated from rice, banana and clivia rot plants show great virulence differentials. BMC Microbiol. 18:136. doi: 10.1186/s12866-018-1300-y

Hudaiberdiev, S., Choudhary, K. S., Alvarez, R. V., Gelencser, Z., Ligeti, B., Lamba, D., et al. (2015). Census of solo LuxR genes in prokaryotic genomes. Front. Cell Infect. Microbiol. 5:20. doi: 10.3389/fcimb.2015.00020

Hughes, D. T., Terekhova, D. A., Liou, L., Hovde, C. J., and Sahl, J. W. (2010). Chemical sensing in mammalian host-bacterial commensal associations. Proc. Natl. Acad. Sci. U.S.A. 107, 9831-9836. doi: 10.1073/pnas.1002551107

Hugouvieux-Cotte-Pattat, N., Condemine, G., and Shevchik, V. E. (2014). Bacterial pectate lyases, structural and functional diversity. Env. Microbiol. Rep. 6, 427440. doi: 10.1111/1758-2229.12166

Hussain, M. B. B. M., Zhang, H., Xu, J., Liu, Q., and Jiang, Z. (2008). The acylhomoserine lactone-type quorum-sensing system modulates cell motility and virulence of Erwinia chrysanthemi pv. zeae. J. Bacteriol. 190, 1045-1053. doi: 10.1128/JB.01472-07

Igarashi, K., and Kashiwagi, K. (2000). Polyamines: mysterious modulators of cellular functions. Biochem. Bioph. Res. Commun. 271, 559-564. doi: 10.1006/ bbrc. 2000.2601

Joshi, J. R., Burdman, S., Lipsky, A., Yariv, S., and Yedidia, I. (2016). Plant phenolic acids affect the virulence of Pectobacterium aroidearum and $P$. carotovorum ssp. brasiliense via quorum sensing regulation. Mol. Plant Pathol. 17, 487-500. doi: $10.1111 / \mathrm{mpp} .12295$

Karatan, E., Duncan, T. R., and Watnick, P. I. (2005). NspS, a predicted polyamine sensor, mediates activation of Vibrio cholerae Biofilm formation by norspermidine. J. Bacteriol. 187, 7434-7443. doi: 10.1128/JB.187.21.7434-7443. 2005

Koski, P., and Vaara, M. (1991). Polyamines as constituents of the outer membranes of Escherichia coli and Salmonella typhimurium. J. Bacteriol. 173, 3695-3699. doi: 10.1128/JB.173.12.3695-3699.1991

Kurihara, S., Sakai, Y., Suzuki, H., Muth, A., Phanstiel, O., and Rather, P. (2013). Putrescine importer PlaP contributes to swarming motility and urothelial cell invasion in Proteus mirabilis. J. Biol. Chem. 288, 15668-15676. doi: 10.1074/jbc. M113.454090

Kurihara, S., Suzuki, H., Oshida, M., and Benno, Y. (2011). A novel putrescine importer required for type 1 Pili-driven surface motility induced by extracellular putrescine in Escherichia coli K-12. J. Biol. Chem. 286, 1018510192. doi: 10.1074/jbc.M110.176032

Lee, J., Sperandio, V., Frantz, D. E., Longgood, J., and Camilli, A. (2009). An alternative polyamine biosynthetic pathway is widespread in bacteria and essential for biofilm formation in Vibrio cholerae. J. Biol. Chem. 284, 9899-9907. doi: 10.1074/jbc.M900110200

Lequette, Y., Lee, J. H., Ledgham, F., Lazdunski, A., and Greenberg, E. P. (2006). A distinct QscR regulon in the Pseudomonas aeruginosa quorum-sensing circuit. J. Bacteriol. 188, 3365-3370. doi: 10.1128/JB.188.9.3365-3370.2006

Liang, Z., Huang, L., He, F., Zhou, X., Shi, Z., Zhou, J., et al. (2019). A SubstrateActivated efflux pump, DesABC, confers zeamine resistance to Dickeya zeae. mBio 10, e00713-19. doi: 10.1128/mBio.00713-19

Liao, L., Zhou, J., Wang, H., He, F., Liu, S., Jiang, Z., et al. (2015). Control of litchi downy blight by zeamines produced by Dickeya zeae. Sci. Rep. 5:15719. doi: 10.1038/srep15719

Liu, H., Coulthurst, S. J., Pritchard, L., Hedley, P. E., Ravensdale, M., Humphris, S., et al. (2008). Quorum sensing coordinates brute force and stealth modes of infection in the plant pathogen Pectobacterium atrosepticum. PLoS Pathog. 4:e1000093. doi: 10.1371/journal.ppat.1000093

Lv, M. (2018). Identification and Characterization of the Virulence Regulatory Mechanisms in Dickeya Zeae EC1. Master's thesis. Guangzhou: South China Agricultural University.

Lv, M., Chen, Y., Hu, M., Yu, Q., Duan, C., Ye, S., et al. (2021). OhrR is a central transcriptional regulator of virulence in Dickeya zeae. Mol. Plant Pathol. 23, 45-59. doi: 10.1111/mpp.13141

Lv, M., Chen, Y., Liao, L., Liang, Z., Shi, Z., Tang, Y., et al. (2018). Fis is a global regulator critical for modulation of virulence factor production and pathogenicity of Dickeya zeae. Sci. Rep. 8:341. doi: 10.1038/s41598-017-18578-2
Lv, M., Hu, M., Li, P., Jiang, Z., and Zhang, L. (2019). A two-component regulatory system VfmIH modulates multiple virulence traits in Dickeya zeae. Mol. Microbiol. 111, 1493-1509. doi: 10.1111/mmi.14233

Mansfield, J., Genin, S., Magori, S., Citovsky, V., and Sriariyanum, M. (2012). Top 10 plant pathogenic bacteria in molecular plant pathology. Mol. Plant Pathol. 13, 614-629. doi: 10.1111/j.1364-3703.2012.00804.x

Mathesius, U., Mulders, S., Gao, M., Teplitski, M., Caetano-Anolles, G., Rolfe, B. G., et al. (2003). Extensive and specific responses of a eukaryote to bacterial quorum-sensing signals. Proc. Natl. Acad. Sci. U.S.A. 100, 1444-1449. doi: 10. 1073/pnas.262672599

Mosquito, S., Meng, X., Devescovi, G., Bertani, I., Geller, A. M., Levy, A., et al. (2020). LuxR solos in the plant endophyte Kosakonia sp. Strain KO348. Appl. Environ. Microb. 86, e00622-20. doi: 10.1128/AEM.00622-20

Nasser, W., Bouillant, M. L., Salmond, G., and Reverchon, S. (1998). Characterization of the Erwinia chrysanthemi expl-expR locus directing the synthesis of two N-acyl-homoserine lactone signal molecules. Mol. Microbiol. 29, 1391-1405. doi: 10.1046/j.1365-2958.1998.01022.x

Nasser, W., Dorel, C., Wawrzyniak, J., Van Gijsegem, F., and Groleau, M. (2013). Vfm a new quorum sensing system controls the virulence of Dickeya dadantii. Environ. Microbiol. 15, 865-880. doi: 10.1111/1462-2920.12049

OrtÍz-Castro, R., Martinez-Trujillo, M., and Lopez-Bucio, J. (2008). N-acyl-Lhomoserine lactones: a class of bacterial quorum-sensing signals alter postembryonic root development in Arabidopsis thaliana. Plant Cell Environ. 31, 1497-1509. doi: 10.1111/j.1365-3040.2008.01863.x

Parkinson, N., DeVos, P., Pirhonen, M., and Elphinstone, J. (2014). Dickeya aquatica sp nov., Isolated from waterways. Int. J. Syst. Evol. Microbial. 64, 2264-2266. doi: 10.1099/ijs.0.058693-0

Patel, C. N., Wortham, B. W., Lines, J. L., Fetherston, J. D., and Perry, R. D. (2006). Polyamines are essential for the formation of plague biofilm. J. Bacteriol. 188, 2355-2363. doi: 10.1128/JB.188.7.2355-2363.2006

Pédron, J., Chapelle, E., Alunni, B., and Van Gijsegem, F. (2018). Transcriptome analysis of the Dickeya dadantii PecS regulon during the early stages of interaction with Arabidopsis thaliana. Mol. Plant Pathol. 19, 647-663. doi: 10. 1111/mpp.12549

Potrykus, M., Golanowska, M., Hugouvieux-Cotte-Pattat, N., and Lojkowska, E. (2014). Regulators involved in Dickeya solani virulence, genetic conservation, and functional variability. Mol. Plant Microbe Interact. 27, 700-711. doi: 10. 1094/MPMI-09-13-0270-R

Potrykus, M., Hugouvieux-Cotte-Pattat, N., and Lojkowska, E. (2018). Interplay of classic Exp and specific Vfm quorum sensing systems on the phenotypic features of Dickeya solani strains exhibiting different virulence levels. Mol. Plant Pathol. 19, 1238-1251. doi: 10.1111/mpp.12614

Reverchon, S., Bouillant, M. L., Salmond, G., and Nasser, W. (1998). Integration of the quorum-sensing system in the regulatory networks controlling virulence factor synthesis in Erwinia chrysanthemi. Mol. Microbiol. 29, 1407-1418. doi: 10.1046/j.1365-2958.1998.01023.x

Samson, R., Legendre, J. B., Christen, R., Fischer-Le Saux, M., Achouak, W., and Garden, L. (2005). Transfer of Pectobacterium chrysanthemi (Burkholder et al. 1953) Brenner et al. 1973 and Brenneria paradisiaca to the genus Dickeya gen. Nov as Dickeya chrysanthemi comb. Nov and Dickeya paradisiaca comb. Nov and delineation of four novel species, Dickeya dadantii sp nov., Dickeya dianthicola sp nov., Dickeya dieffenbachiae sp nov and Dickeya zeae sp nov. Int. J. Syst. Evol. Microbiol. 55, 1415-1427. doi: 10.1099/ijs.0.02791-0

Schenk, S. T., Stein, E., Kogel, K. H., and Schikora, A. (2012). Arabidopsis growth and defense are modulated by bacterial quorum sensing molecules. Plant Signal. Behav. 7, 178-181. doi: 10.4161/psb.18789

Schikora, A., Schenk, S. T., Stein, E., Molitor, A., Zuccaro, A., and Kogel, K. H. (2011). N-acyl-homoserine lactone confers resistance toward biotrophic and hemi biotrophic pathogens via altered activation of AtMPK6. Plant Physiol. 157, 1407-1418. doi: 10.1104/pp.111.180604

Schuhegger, R., Ihring, A., Gantner, S., Bahnweg, G., Knappe, C., Vogg, G., et al. (2006). Induction of systemic resistance in tomato by $\mathrm{N}$-acyl-L-homoserine lactone-producing rhizosphere bacteria. Plant Cell Environ. 29, 909-918. doi: 10.1111/j.1365-3040.2005.01471.x

Shi, Z., Wang, Q., Li, Y., Liang, Z., Xu, L., Zhou, J., et al. (2019). Putrescine is an intraspecies and interkingdom Cell-Cell communication signal modulating the virulence of Dickeya zeae. Front. Microbiol. 10:1950. doi: 10.3389/fmicb.2019. 01950 
Sturgill, G., and Rather, P. N. (2004). Evidence that putrescine acts as an extracellular signal required for swarming in Proteus mirabilis. Mol. Microbiol. 51, 437-446. doi: 10.1046/j.1365-2958.2003.03835.x

Tang, Y. X. (2017). Functional Analysis of Polyamine Transporter Signaling Pathway in Dickeya zeae MS3. Master's thesis. Guangzhou: South China Agricultural University.

van der Merwe, J. J., Coutinho, T. A., Korsten, L., and van der Waals, J. E. (2010). Pectobacterium carotovorum subsp brasiliensis causing blackleg on potatoes in South Africa. Eur. J. Plant Pathol. 126, 175-185. doi: 10.1007/s10658009-9531-2

van der Wolf, J. M., Nijhuis, E. H., Kowalewska, M. J., Saddler, G. S., and Parkinson, N. (2014). Dickeya solani sp nov., A pectinolytic plant-pathogenic bacterium isolated from potato (Solanum tuberosum). Int. J. Syst. Evol. Microbiol. 64, 768-774. doi: 10.1099/ijs.0.052944-0

von Rad, U., Klein, I., Dobrev, P. I., Kottova, J., and Zazimalova, E. (2008). Response of Arabidopsis thaliana to N-hexanoyl-DL-homoserine-lactone, a bacterial quorum sensing molecule produced in the rhizosphere. Planta 229, 73-85. doi: 10.1007/s00425-008-0811-4

Wortham, B. W., Oliveira, M. A., Fetherston, J. D., and Perry, R. D. (2010). Polyamines are required for the expression of key Hms proteins important for Yersinia pestis biofilm formation. Environ. Microbiol. 12, 2034-2047. doi: 10.1111/j.1462-2920.2010.02219

Yang, C. H., Gavilanes-Ruiz, M., Okinaka, Y., Vedel, R., and Berthuy, I. (2002). Hrp genes of Erwinia chrysanthemi 3937 are important virulence factors. Mol. Plant Microbe Interact. 15, 472-480. doi: 10.1094/MPMI.2002.15.5. 472

Yang, S. H., Perna, N. T., Cooksey, D. A., Okinaka, Y., and Lindow, S. E. (2004). Genome-wide identification of plant-upregulated genes of Erwinia chrysanthemi 3937 using a GFP-Based IVET leaf array. Mol. Plant Microbe Interact. 17, 999-1008. doi: 10.1094/MPMI.2004.17.9.999

Yap, M. N., Yang, C. H., Barak, J. D., Jahn, C. E., and Charkowski, A. O. (2005). The Erwinia chrysanthemi type III secretion system is required for multicellular behavior. J. Bacteriol. 187, 639-648. doi: 10.1128/JB.187.2.639-648. 2005

Zhang, L., Jia, Y., Wang, L., and Fang, R. (2007). A proline iminopeptidase gene upregulated in planta by a LuxR homologue is essential for pathogenicity of
Xanthomonas campestris pv. campestris. Mol. Microbiol. 65, 121-136. doi: 10. 1111/j.1365-2958.2007.05775.x

Zhong, J. (2014). The Roles of ExpR/ExpI in Regulation of Dickeya zeae Pathogenesis. Ph.D. thesis. Guangzhou: South China Agricultural University.

Zhou, J., Cheng, Y., Lv, M., Liao, L., Chen, Y., Gu, Y., et al. (2015). The complete genome sequence of Dickeya zeae EC1 reveals substantial divergence from other Dickeya strains and species. BMC Genomics 16:571. doi: 10.1186/s12864-0151545-x

Zhou, J., Zhang, H., Lv, M., Chen, Y., Liao, L., Chang, Y., et al. (2016). SlyA regulates phytotoxin production and virulence in Dickeya zeae EC1. Mol. Plant Pathol. 17, 1398-1408. doi: 10.1111/mpp.12376

Zhou, J., Zhang, H., Wu, J., Liu, Q., Xi, P., Lee, J., et al. (2011). A novel multidomain polyketide synthase is essential for zeamine production and the virulence of Dickeya zeae. Mol. Plant Microbe Interact. 24, 1156-1164. doi: 10.1094/MPMI04-11-0087

Zhou, L., Wang, J., and Zhang, L. (2007). Modulation of bacterial type III secretion system by a spermidine transporter dependent signaling pathway. PLoS One 2:e1291. doi: 10.1371/journal.pone.0001291

Conflict of Interest: The authors declare that the research was conducted in the absence of any commercial or financial relationships that could be construed as a potential conflict of interest.

Publisher's Note: All claims expressed in this article are solely those of the authors and do not necessarily represent those of their affiliated organizations, or those of the publisher, the editors and the reviewers. Any product that may be evaluated in this article, or claim that may be made by its manufacturer, is not guaranteed or endorsed by the publisher.

Copyright (c) $2022 \mathrm{Liu}, \mathrm{Hu}$, Zhang, Xue, Chen, Hu, Zhang and Zhou. This is an open-access article distributed under the terms of the Creative Commons Attribution License (CC BY). The use, distribution or reproduction in other forums is permitted, provided the original author(s) and the copyright owner(s) are credited and that the original publication in this journal is cited, in accordance with accepted academic practice. No use, distribution or reproduction is permitted which does not comply with these terms. 